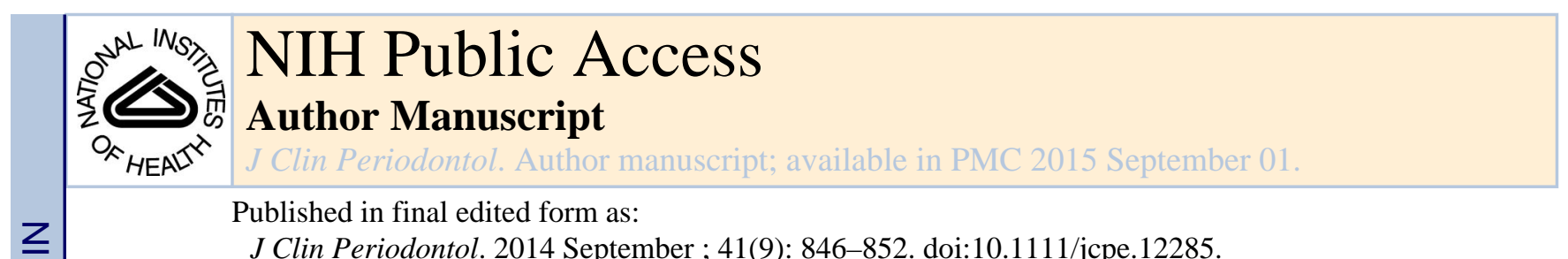

J Clin Periodontol. 2014 September ; 41(9): 846-852. doi:10.1111/jcpe.12285.

\title{
Assessment of Partial-Mouth Periodontal Examination Protocols for Periodontitis Surveillance
}

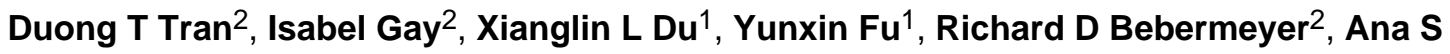 \\ Neumann ${ }^{2}$, Charles Streckfus ${ }^{2}$, Wenyaw Chan ${ }^{1}$, and Muhammad F Walji ${ }^{2,3}$ \\ 1 School of Public Health, The University of Texas Health Science Center at Houston, USA \\ ${ }^{2}$ School of Dentistry, The University of Texas Health Science Center at Houston, USA \\ ${ }^{3}$ School of Biomedical Informatics, The University of Texas Health Science Center at Houston, \\ USA
}

\section{Abstract}

Objective-To evaluate bias associated with nine identified partial-mouth periodontal examination (PMPE) protocols in estimating periodontitis prevalence using the periodontitis case definition given by the Centers of Disease Control and Prevention and American Academy of Periodontology (CDC/AAP).

Material and Methods-Prevalence from full-mouth examination was determined in a sample of 3,667 adults $\geq 30$ years old from the National Health and Nutrition Examination Survey (NHANES) 2009-2010. Prevalence, absolute bias, relative bias, sensitivity and inflation factor were derived for these protocols according to the CDC/AAP definition and half-reduced CDC/AAP definition as $\mathbf{5 0 \%}$ of sites were measured.

Results-Bias in moderate and severe periodontitis prevalence ranged between $11.1 \%$ to $52.5 \%$ and $27.1 \%$ to $76.3 \%$ for full-mouth mesiobuccal-distolingual protocol and half-mouth mesiobuccal protocol respectively; according to the CDC/AAP definition. With half-reduced CDC/AAP definition, half-mouth four sites protocol provided small absolute bias (3.2\%) and relative bias $(9.3 \%)$ for the estimates of moderate periodontitis prevalence; corresponding biases for severe periodontitis were $-1.2 \%$ and $-10.2 \%$.

Conclusion-Periodontitis prevalence can be estimated with limited bias when a half-mouth four sites protocol and a half-reduced CDC/AAP case definition are used in combination.

\section{Keywords}

Periodontitis; surveillance; partial mouth periodontal examination protocols; CDC/AAP case definition of periodontitis

Corresponding author: Duong T. Tran DDS, MPH, PhD The University of Texas Health Science Center at Houston School of Dentistry 7500 Cambridge St. Houston, TX 77054 Tel: 713-486-4583 Duong.T.Tran@uth.tmc.edu. 


\section{Introduction}

Periodontitis remains as a critical disease with potential systemic implications which affects worldwide populations (Eke et al., 2012a, Petersen and Ogawa, 2012). Various surveillance programs have implemented the use of different methods to measure periodontal disease in populations (Dye and Thornton-Evans, 2007, Tu et al., 2013, Eke et al., 2012a, White et al., 2012, Gokalp et al., 2010). However, researchers have not reached a consensus on an effective method due to various levels of underestimation of periodontitis prevalence (Susin et al., 2005, Eke et al., 2010, Vettore et al., 2007, Leroy et al., 2010).

Full-mouth periodontal examination (FMPE) protocol is the gold standard to determine an individual periodontal status. It has been implemented in the National Health and Nutrition Examination Survey (NHANES) since 2009 (Centers for Disease Control and Prevention, 2013b) to evaluate the trend or change in periodontitis prevalence in the adult U.S. population (Healthy People 2020). While a comprehensive examination is feasible as part of routine dental care; FMPE that includes evaluation of 168 sites (six sites per tooth) is labor, time and resource demanding for periodontitis surveillance (Kingman et al., 2008). Although full-mouth periodontal examination was allotted less than five minutes in the NHANES 2009-2010 (Centers for Disease Control and Prevention, 2013a), a study showed that FMPE took an average of 28.8 minutes to measure a complete periodontal exam (Owens et al., 2003).

Therefore, partial-mouth periodontal examination (PMPE) protocols which assess fewer sites yet, still estimating the overall periodontal status have been used. PMPE definition is the clinical assessment of representative sets of teeth or probing sites (Kingman and Albandar, 2002). Accuracy of PMPE protocols in determining true prevalence was shown to vary by disease level in population, periodontitis case definition, tooth location, number and probing sites (Susin et al., 2005, Eke et al., 2010).

In 2007, the CDC and American Academy of Periodontology (CDC/AAP) introduced a case definition of periodontitis to be used for population-based surveillance (Eke et al., 2012b). According to this definition, two PMPE protocols used in the NHANES III and NHANES 1999-2004 provided high levels of underestimation of periodontitis prevalence (Eke et al., 2012a, Eke et al., 2010). However, the application of the same CDC/AAP case definition of periodontitis to both, FMPE and PMPE protocols may cause bias in PMPE protocols estimates of prevalence. Since PMPE examined fewer probing sites than FMPE protocols, the use of a FMPE case definition applied to PMPE protocol would be inaccurate; i.e., the CDC/AAP definition of periodontitis should be different in FMPE and PMPE protocols. This source of bias has not been previously addressed and is critical to establish the true prevalence of periodontitis in a population.

There has been no evaluation of the accuracy of PMPE protocols, which were previously identified as the ones with the lowest bias or frequent use (Susin et al., 2005, Tran et al., 2013), as compared to FMPE when assessing prevalence of periodontitis using the CDC/AAP definition. It is important to have data on bias for a wide range of PMPE protocols according to this definition to assess the amount of the underestimation when 
comparing prevalence of periodontitis within and between countries. Hence, our specific aim is to evaluate bias for different PMPE protocols in estimating periodontitis prevalence according to the CDC/AAP case definition and our modified half-reduced CDC/AAP case definition in the NHANES 2009-2010 dataset.

\section{Material and Methods}

\section{Partial-mouth periodontal examination protocols and data origin}

PMPE protocols performance was evaluated using existent data from the NHANES 2009-2010 (Centers for Disease Control and Prevention, 2013b). Nine PMPE protocols previously identified as the ones with the lowest bias or frequent use (Susin et al., 2005, Tran et al., 2013) were assessed in this study. As measurements on mid-buccal and midlingual sites are not used in the CDC/AAP case definition, these sites were excluded from the nine PMPE protocols. The protocols were: 1) mesiobuccal (MB), distobuccal (DB), mesiolingual (ML), and distolingual (DL) measurements on six "Ramfjord" teeth maxillary right and mandibular left first molars, maxillary left and mandibular right first premolars, and maxillary right and mandibular left central incisors; 2) MB-DB-ML-DL measurements on ten teeth of the Community Periodontal Index (CPI protocol) - maxillary and mandibular first and second molars, maxillary right and mandibular left central incisors; 3) MB measurements on all teeth; 4) MB-DB measurements on all teeth; 5) MB-DL measurements on all teeth; 6) MB measurements on teeth on random half-mouth, a maxillary and mandibular quadrant randomly selected; 7) random half-mouth MB-DB; 8) random half-mouth MB-DL; and 9) random half-mouth MB-DB-ML-DL (also named as half-mouth 4 sites). The randomness of the selected quadrant for each subject was created using Stata's random-number function.

NHANES 2009-2010 is a representative sample of the U.S. population including 5,037 adults aged $\geq 30$ years for periodontal examination (Centers for Disease Control and Prevention, 2013b). From the original dataset, we applied participant exclusion criteria as shown in Figure 1. Subjects with less than six teeth were excluded as several PMPE protocols had missing values for these subjects. 3,667 subjects were included in the evaluation of the PMPE protocols.

NHANES participants were examined by dental hygienists with North Carolina (3-6-9-12-15) Periodontal Probe (Hu-Friedy, Chicago, IL, USA) at mobile examination centers. Each measurement is rounded to the lowest whole millimeter. Periodontal measurements were performed at six sites per tooth in all dentition. Calibration of examiners for NHANES 2009-2010 subjects was described (Centers for Disease Control and Prevention, 2013b) but the inter-rater agreement has not been published. The latest calibration information for periodontitis published was for NHANES 2003-2004 with kappa scores between 0.64 and 0.82 (Dye et al., 2008).

\section{Data analyses}

We compared periodontitis prevalence determined by FMPE with the prevalence determined by PMPE protocols using the same data. CDC/AAP (Eke et al., 2012b) defined periodontitis 
as follows: Mild: $\geq 2$ interproximal sites with $\geq 3 \mathrm{~mm} \mathrm{CAL}$, and $\geq 2$ interproximal sites with $\geq 4 \mathrm{~mm}$ PD (on different teeth) or 1 site with $\geq 5 \mathrm{~mm}$ PD; Moderate: $\geq 2$ interproximal sites with $\geq 4 \mathrm{~mm}$ CAL (on different teeth), or $\geq 2$ interproximal sites with $\geq 5 \mathrm{~mm}$ PD (on different teeth); Severe: $\geq 2$ interproximal sites with $\searrow 6 \mathrm{~mm} \mathrm{CAL}$ (on different teeth) and $\geq 1$ interproximal site with $\geq 5 \mathrm{~mm}$ PD (on the same site with CAL $\succeq 6 \mathrm{~mm}$ or on different sites).

Because the PMPE protocols examined $<50 \%$ of total sites, we suggested a half-reduced CDC/AAP case definition for the PMPE protocols which is defined as Mild: $\geq 1$ interproximal sites with $\geq 3 \mathrm{~mm} \mathrm{CAL}$, and $\geq 1$ interproximal sites with $\geq 4 \mathrm{~mm}$ PD (on the same site with CAL $\geq 3 \mathrm{~mm}$ or on different sites) or 1 site with $\geq 5 \mathrm{~mm}$ PD; Moderate: $\geq 1$ interproximal sites with $\geq 4 \mathrm{~mm} \mathrm{CAL}$, or $\geq 1$ interproximal sites with $\geq 5 \mathrm{~mm}$ PD; Severe: $\geq 1$ interproximal sites with $\geq 6 \mathrm{~mm} \mathrm{CAL}$ and $\geq 1$ interproximal site with $\geq 5 \mathrm{~mm}$ PD (on the same site with CAL $₫ 6 \mathrm{~mm}$ or on different sites). In this research, we have chosen to evaluate only moderate and severe periodontitis categories to eliminate a potential risk on the bias for PMPE protocols in mild periodontitis prevalence.

The comparison was conducted by: 1) applying the half-reduced CDC/AAP definition to the PMPE protocols and 2) applying the CDC/AAP definition to the PMPE protocols. The CDC/AAP definition was always applied to the FMPE protocol.

The comparison was based on absolute bias, relative bias, sensitivity, and inflation factor:

$$
\begin{aligned}
\text { Absolute bias } & =\text { prevalence }_{P M P E}-\text { prevalence }_{F M P E} \\
\text { Relative bias } & =\frac{\text { prevalence } \left._{P M P E}-\text { prevalence }_{F M P E}\right)}{\text { prevalence }_{F M P E} \times 100} \\
\text { Sensitivity } & =\frac{\text { prevalence }_{P M P E} \times 100}{\text { prevalence }_{F M P E}} \\
\text { Inflation factor } & =\frac{\text { prevalence }_{F M P E} \times 100}{\text { prevalence }_{P M P E}}
\end{aligned}
$$

In case two definitions were applied to FMPE and PMPE protocol, PMPE protocol may overestimate the periodontitis prevalence, resulting a positive relative bias and an inflation factor $<100 \%$. For the NHANEs sample, PMPE protocol associated bias for prevalence estimate was stratified by age groups at 10-year intervals, race/ethnicity, gender, education level, and number of teeth categorized by median. These variables affect periodontitis prevalence (Burt, 2005) which was related with bias for the estimates provided by PMPE protocols (Kingman et al., 1988, Susin et al., 2005). Patients with missing data of any variable above were not included in the subgroup analysis for that variable. Data analysis was conducted using STATA 13 (StataCorp, 2013).

\section{Results Subjects}

For the NHANES sample, age ranged between 30 to 80 years. Approximately half of subjects were male, white, having some college education or higher. Mean number of teeth was 23.4, median was 25 teeth, and the range was 6 to 28 teeth. 13.6\% of sites had PD $\geq 3$ $\mathrm{mm}$ and $19.6 \%$ of sites had CAL $\geq 3 \mathrm{~mm}$ (Table 1 ). 


\section{Accuracy of the PMPE protocols in the NHANES sample}

CDC/AAP case definition applied to the PMPE protocols-According to the

CDC/AAP case definition, the prevalence of moderate and severe periodontitis was $34.3 \%$

and $11.8 \%$ respectively from full-mouth examination. The PMPE protocols provided various estimates of prevalence, absolute bias, relative bias, sensitivities and inflation factor. The prevalence estimated by the PMPE protocols varied from $16.3 \%$ to $30.5 \%$ for moderate periodontitis and from $2.9 \%$ to $8.6 \%$ for severe periodontitis. The underestimation level for PMPE protocols ranged from $11.1 \%$ (full-mouth MB-DL) to $52.5 \%$ (half-mouth MB) for moderate periodontitis prevalence, and from $27.1 \%$ (full-mouth MB-DL) to $76.3 \%$ (halfmouth MB) for severe periodontitis prevalence. The full-mouth MB-DL protocol provided the smallest absolute bias for moderate periodontitis $(-3.8 \%)$ and for severe periodontitis $(-3.2 \%)$ while half-mouth MB protocol provided the largest absolute biases (-18\% and $-9 \%$ for moderate and severe disease respectively). The range of sensitivity was $23.7 \%$ to $88.9 \%$ and of inflation factor was $112.5 \%$ to $421.4 \%$ (Table 2).

Half-reduced CDC/AAP case definition applied to the PMPE protocols-Biases and inflation factors for all PMPE protocols decreased remarkably when we applied the halfreduced CDC/AAP case definition to the PMPE protocols. Among all nine PMPE protocols evaluated, the CPI, full-mouth MB-DB, full-mouth MB-DL, and half-mouth four sites protocols provided smaller absolute biases (-1.6\% to $0.6 \%)$ and relative biases (-13.6\% to $5.1 \%$ ) for severe periodontitis; their corresponding biases for moderate periodontitis were $3.2 \%$ to $5.2 \%$ and $9.3 \%$ to $15.2 \%$. Inflation factors for these four protocols were close to $100 \%$ (Table 3).

Effect of age, gender, education and number of teeth on bias-Table 4 and Appendix 1 present the effect of age, gender, education and number of teeth on bias associated with the CPI, full-mouth MB-DB, full-mouth MB-DL, and half-mouth four sites protocols for prevalence estimates according to the half-reduced CDC/AAP case definition. Periodontitis prevalence increased with age, male gender, lower education level, and lower number of teeth. The half-mouth four sites protocol provided absolute bias ( $0.8 \%$ to $5.6 \%)$ and relative bias ( $2 \%$ to $18.3 \%$ ) for moderate periodontitis across all strata. Regarding severe periodontitis, absolute bias for half-mouth four sites protocol ranged from $-2.9 \%$ to $0.8 \%$ and associated relative bias ranged from $-22.5 \%$ to $22.9 \%$. Other PMPE protocols provided relative bias $<21 \%$ in all strata except age groups 30-39 years, $40-49$ years and group with more than 25 teeth. The severe periodontitis estimate had relative bias $57.1 \%$ based on the CPI protocol and $40 \%$ based on the full-mouth MB-DL protocol in the age group 30-39 years.

\section{Discussion}

This study presented the differences between nine PMPE protocols and the FMPE in two situations: 1) using the same CDC/AAP definition for the PMPE protocols and 2) using the half-reduced CDC/AAP definition for the PMPE protocols. In the first situation, although the full-mouth MB-DL performed better than other protocols, it did not closely estimate severe periodontitis prevalence in the NHANES sample (absolute bias, $-3.2 \%$; relative bias, 
$-27.1 \%$; sensitivity, $72.9 \%$ ). We demonstrated that only with the half-reduced CDC/AAP definition, the half-mouth four sites protocol closely approximated FMPE protocol in estimates of periodontitis prevalence in the NHANES sample. Among the nine PMPE protocols evaluated, it provided small absolute bias (3.2\%) and relative bias $(9.3 \%)$ for the estimates of moderate periodontitis prevalence; corresponding biases for severe periodontitis were $-1.2 \%$ and $-10.2 \%$. It also consistently provided small absolute bias $(0.8 \%$ to $5.6 \%)$ and relative bias ( $2 \%$ to $18.3 \%$ ) for moderate periodontitis across all strata of age, gender, race/ ethnicity, education level, and number of teeth; corresponding biases for severe periodontitis were $-2.9 \%$ to $0.8 \%$ and $-22.5 \%$ to $22.9 \%$.

We suggest using the half-mouth four sites protocol instead of the FMPE for populationbased surveillance of periodontitis. By probing only 56 sites, it has important implications for reduced examination time in periodontitis surveillance. In addition to small absolute bias, we can infer from a study (Nelson et al., 2001) that a PMPE protocol with a relative bias $\leq 20 \%$ was considered to have an acceptable performance as relative bias equal to sensitivity minus one (Kingman et al., 1988). Although the half-mouth four sites protocol provided a relative bias of 22.9\% for severe periodontitis in age group 30-39 years and $-22.5 \%$ in age group 70-80 years in accordance with half-reduced CDC/AAP definition, the corresponding absolute biases for these two age groups were small $(0.8 \%$ and $-2.9 \%)$.

We also suggest using the newly proposed CDC/AAP case definition for the half-mouth four sites protocol because periodontitis case definition strongly impacted performance of PMPE protocols. Bias for the PMPE protocols in estimates of periodontitis prevalence decreased significantly with the application of the half-reduced CDC/AAP definition, compared to the application of full CDC/AAP definition. Previous studies showed that the half-mouth MBDB, half-mouth MB-DL, half-mouth MB-DB-DL, half-mouth MB-DB-ML, and half-mouth four sites protocols largely underestimated periodontitis prevalence (Eke et al., 2010, Eke et al., 2012a). However, these studies applied the CDC/AAP definition to the PMPE protocols which might contribute partly to the underestimation. This study is the first to provide evidence of bias caused by application of the same CDC/AAP definition to both, FMPE and PMPE protocols.

With the half-reduced CDC/AAP case definition, 40 sites CPI protocol substantially overestimated periodontitis prevalence in the age group 30-39 years and in group of subjects with $\geq 25$ teeth in the NHANES sample. The bias could be partly attributed to the protocol design and to the low prevalence of severe periodontitis in these two groups, $3.5 \%$ and $5.8 \%$, respectively. It was shown that bias was influenced by the number and location of sites assessed (Eke et al., 2010, Susin et al., 2005) and that relative bias increased when disease prevalence decreased (Kingman et al., 1988). The poor performance of the CPI protocol in these two groups limits its use in periodontitis surveillance although it also performed well in other strata.

The full-mouth MB-DB performance was acceptable in accordance with the half-reduced CDC/AAP case definition. However, it provided higher bias compared to half-mouth four sites protocol at various levels across all strata. Although they both examine 56 sites, it might be easier and faster to examine only buccal sites. If researchers prefer accuracy, half- 
mouth four sites protocol would be the best choice. The advantage of this study was the evaluation of the PMPE protocols performance in the NHANES 2009-2010 sample representing the U.S. adult population. Our results can be applied to studies that examine the general population. Additionally, it would be ideal to validate the best performed PMPE protocols in other population-based surveillance using FMPE such as NHANES 2011-2012. The disadvantage of our study was the inability to assess the inter-rater agreement between examiners for periodontal measurements in the NHANES 2009-2010 as it was not published.

\section{Conclusion}

PMPE protocols associated biases varied with the number and sites of the protocol, disease level in population, and periodontitis case definition. Our findings suggest that the prevalence of periodontitis can be estimated with limited bias when a half-mouth four sites protocol and a half-reduced CDC/AAP case definition are used in combination.

\section{Supplementary Material}

Refer to Web version on PubMed Central for supplementary material.

\section{Acknowledgements}

This project is supported in part by the NLM G08LM010075 grant. We wish to thank Krishna Kumar Kookal at UTSOD, Houston, TX.

\section{References}

Burt B. Position paper: epidemiology of periodontal diseases. Journal of Periodontology. 2005; 76:1406-1419. [PubMed: 16101377]

Centers for Disease Control and Prevention. Mobile Exam Center Components Descriptions[Online]. Centers for Disease Control and Prevention; Atlanta: 2013a. Available: http://www.cdc.gov/nchs/ data/nhanes/nhanes_09_10/meccomp_f.pdf [March 19th 2014]

Centers for Disease Control and Prevention. National Health and Nutrition Examination Survey[Online]. Centers for Disease Control and Prevention; Atlanta: 2013b. Available: http:// www.cdc.gov/nchs/nhanes/nhanes2009-2010/OHXPER_F.htm [July 6th 2013]

Dye BA, Nowjack-Raymer R, Barker LK, Nunn JH, Steele JG, Tan S, Lewis BG, Beltran-Aguilar ED. Overview and quality assurance for the oral health component of the National Health and Nutrition Examination Survey (NHANES), 2003-04. Journal of Public Health Dentistry. 2008; 68:218-226. [PubMed: 18248340]

Dye BA, Thornton-Evans G. A brief history of national surveillance efforts for periodontal disease in the United States. Journal of Periodontology. 2007; 78:1373-1379. [PubMed: 17608609]

Eke PI, Dye BA, Wei L, Thornton-Evans GO, Genco RJ. Prevalence of periodontitis in adults in the United States: 2009 and 2010. Journal of Dental Research. 2012a; 91:914-920. [PubMed: 22935673]

Eke PI, Page RC, Wei L, Thornton-Evans G, Genco RJ. Update of the case definitions for populationbased surveillance of periodontitis. Journal of Periodontology. 2012b; 83:1445-1454.

Eke PI, Thornton-Evans GO, Wei L, Borgnakke WS, Dye BA. Accuracy of NHANES periodontal examination protocols. Journal of Dental Research. 2010; 89:1208-1213. [PubMed: 20858782]

Gokalp SG, Dogan BG, Tekcicek MT, Berberoglu A, Unluer S. National survey of oral health status of children and adults in Turkey. Community Dent Health. 2010; 27:12-17. [PubMed: 20426255] 
Healthy People. Improving the Health of Americans [Online]. U.S. Department of Health and Human Services; Washington DC: 2020. Available: http://www.healthypeople.gov/2020/ topicsobjectives2020/objectiveslist.aspx?topicId=32 [October 3rd 2013]

Kingman A, Albandar JM. Methodological aspects of epidemiological studies of periodontal diseases. Periodontology. 2002; 29:11-30. 2000.

Kingman A, Morrison E, Loe H, Smith J. Systematic errors in estimating prevalence and severity of periodontal disease. Journal of Periodontology. 1988; 59:707-713. [PubMed: 3264570]

Kingman A, Susin C, Albandar JM. Effect of partial recording protocols on severity estimates of periodontal disease. Journal of Clinical Periodontology. 2008; 35:659-667. [PubMed: 18513337]

Leroy R, Eaton KA, Savage A. Methodological issues in epidemiological studies of periodontitis how can it be improved? BMC Oral Health. 2010; 10:8. [PubMed: 20409298]

Nelson DE, Holtzman D, Bolen J, Stanwyck CA, Mack KA. Reliability and validity of measures from the Behavioral Risk Factor Surveillance System (BRFSS). Soz Praventivmed. 2001; 46(Suppl 1):S3-42. [PubMed: 11851091]

Owens JD, Dowsett SA, Eckert GJ, Zero DT, Kowolik MJ. Partial-mouth assessment of periodontal disease in an adult population of the United States. Journal of Periodontology. 2003; 74:12061213. [PubMed: 14514235]

Petersen PE, Ogawa H. The global burden of periodontal disease: towards integration with chronic disease prevention and control. Periodontology. 2012; 2000; 60:15-39.

Statacorp. Stata Statistical Software: Release 13. StataCorp LP.; College Station, TX: 2013.

Susin C, Kingman A, Albandar JM. Effect of partial recording protocols on estimates of prevalence of periodontal disease. Journal of Periodontology. 2005; 76:262-267. [PubMed: 15974851]

Tran DT, Gay I, Du XL, Fu Y, Bebermeyer RD, Neumann AS, Streckfus C, Chan W, Walji MF. Assessing periodontitis in populations: a systematic review of the validity of partial-mouth examination protocols. Journal of Clinical Periodontology. 2013; 40:1064-1071. [PubMed: 24192071]

Tu Y-K, D'aiuto F, Lin H-J, Chen Y-W, Chien K-L. Relationship between metabolic syndrome and diagnoses of periodontal diseases amongst participants in a large Taiwanese cohort. Journal of Clinical Periodontology. 2013; 40:994-1000. [PubMed: 24007401]

Vettore MV, Lamarca Gde A, Leao AT, Sheiham A, Leal Mdo C. Cad Saude Publica. 2007; Partial recording protocols for periodontal disease assessment in epidemiological surveys.23:33-42. [PubMed: 17187102]

White DA, Tsakos G, Pitts NB, Fuller E, Douglas GV, Murray JJ, Steele JG. Adult Dental Health Survey 2009: common oral health conditions and their impact on the population. British Dental Journal. 2012; 213:567-572. [PubMed: 23222333] 


\section{Clinical Relevance}

Scientific rationale for the study: PMPE protocols are critical to screen populations. This study evaluated bias associated with nine previously identified protocols.

Principal Findings: Bias in moderate and severe periodontitis prevalence ranged between 11.1-52.5\% and 27.1-76.3\% respectively; according to the CDC/AAP definition. With half-reduced CDC/AAP definition, half-mouth four sites protocol provided small absolute bias (3.2\%-1.2\% for moderate and severe periodontitis respectively) and relative bias $(9.3 \%,-10.2 \%)$.

Practical Implications: Half-mouth four sites protocol in accordance with half-reduced CDC/AAP definition is suggested for large population based clinical trials as it closely approximates full mouth examination in estimates of periodontitis prevalence. 


\section{NHANES subjects participated in periodontal examination $(\mathrm{n}=5,037)$}

Excluded $(\mathrm{n}=1,370)$ :

-Edentulous $(\mathrm{n}=341)$

- Health condition that required antibiotic prophylaxis before periodontal probing $(n=295)$

- No periodontal examination done $(n=619)$

- Partial periodontal exam $(\mathrm{n}=41)$

- Less than 6 teeth $(n=74)$

Eligible subjects $(n=3,667)$

Figure 1.

Selection of NHANES 2009-2010 subjects 


\section{Table 1}

Characteristics of the NHANES 2009-2010 sample

\begin{tabular}{lcc}
\hline & \multicolumn{2}{c}{ NHANES } \\
\cline { 2 - 3 } Characteristic & Number & $\%$ \\
\hline Total & 3,667 & \\
Age, $\mathbf{y}$ & & \\
$30-39$ & 877 & 23.9 \\
$40-49$ & 903 & 24.6 \\
$50-59$ & 730 & 19.9 \\
$60-69$ & 629 & 17.2 \\
270 & 528 & 14.4 \\
Gender & & \\
Male & 1,831 & 49.9 \\
Female & 1,836 & 50.1
\end{tabular}

Race/ Ethnicity*

$\begin{array}{lcc}\text { Mexican American } & 663 & 18.1 \\ \text { Other Hispanic } & 398 & 10.9 \\ \text { Non-Hispanic White } & 1,751 & 47.8 \\ \text { Non-Hispanic Black } & 656 & 17.9 \\ \text { Other Race - Including Multi-Racial } & 199 & 5.3\end{array}$

Education level

Less than high school

$991 \quad 27.1$

High school or equivalent

$798 \quad 21.8$

Some college or higher

$1,870 \quad 51.1$

\begin{tabular}{lcc} 
& Mean & SD \\
\hline $\begin{array}{l}\text { Number of teeth present (Minimum-maximum) } \\
\text { \% of sites with PD }\end{array}$ & $23.4(6-28)$ & 5.6 \\
$\geq 3 \mathrm{~mm}$ & 13.6 & \\
$\geq 4 \mathrm{~mm}$ & 5.0 & 18.1 \\
$\geq 5 \mathrm{~mm}$ & 1.7 & 5.5 \\
$\geq 6 \mathrm{~mm}$ & 0.7 & 3.2 \\
\% of sites with CAL & & \\
$\geq 3 \mathrm{~mm}$ & 19.6 & 23.9 \\
$\geq 4 \mathrm{~mm}$ & 10.9 & 18.7 \\
$\geq 5 \mathrm{~mm}$ & 5.9 & 13.5 \\
$\geq 6 \mathrm{~mm}$ & 3.2 & 9.8 \\
\hline
\end{tabular}


Table 3

Accuracy of PMPE protocols in estimating periodontitis prevalence according to half-reduced CDC/AAP case definition in NHANES sample

\begin{tabular}{|c|c|c|c|c|c|c|c|c|}
\hline & \multicolumn{2}{|c|}{ Periodontitis prevalence $(\%)$} & \multicolumn{2}{|c|}{ Absolute bias (\%) } & \multicolumn{2}{|c|}{ Relative bias (\%) } & \multicolumn{2}{|c|}{ Inflation factor $(\%)$} \\
\hline & Moderate PD (SE) & Severe PD (SE) & Moderate PD & Severe PD & Moderate PD & Severe PD & Moderate PD & Severe PD \\
\hline $\mathrm{FMPE}^{*}$ & $34.3(0.8)$ & $11.8(0.5)$ & Ref & Ref & Ref & Ref & Ref & Ref \\
\hline \multicolumn{9}{|c|}{ Full-mouth PMPEs ${ }^{* *}$} \\
\hline Ramfjord & $30.7(0.8)$ & $6.8(0.4)$ & -3.6 & -5.0 & -10.5 & -42.4 & 111.7 & 173.5 \\
\hline CPI & $38.1(0.8)$ & $11.5(0.5)$ & 3.8 & -0.3 & 11.1 & -2.5 & 90.0 & 102.6 \\
\hline MB & $33.9(0.8)$ & $7.5(0.4)$ & -0.4 & -4.3 & -1.2 & -36.4 & 101.2 & 157.3 \\
\hline MB-DB & $39.5(0.8)$ & $10.2(0.5)$ & 5.2 & -1.6 & 15.2 & -13.6 & 86.8 & 115.7 \\
\hline MB-DL & $39.1(0.8)$ & $12.4(0.5)$ & 4.8 & 0.6 & 14.0 & 5.1 & 87.7 & 95.2 \\
\hline \multicolumn{9}{|c|}{ Half-mouth PMPEs ${ }^{* *}$} \\
\hline MB & $27.4(0.7)$ & $5.0(0.4)$ & -6.9 & -6.8 & -20.1 & -57.6 & 125.2 & 236.0 \\
\hline MB-DB & $32.6(0.8)$ & $7.2(0.4)$ & -1.7 & -4.6 & -5.0 & -39.0 & 105.2 & 163.9 \\
\hline MB-DL & $4.6(0.8)$ & $8.4(0.5)$ & -0.3 & -3.4 & -0.9 & -28.8 & 99.1 & 140.5 \\
\hline 4 sites & $37.5(0.8)$ & $10.6(0.5)$ & 3.2 & -1.2 & 9.3 & -10.2 & 91.5 & 111.3 \\
\hline
\end{tabular}

$\mathrm{PD}=$ periodontitis

Negative and positive biases mean underestimation and overestimation of periodontitis prevalence, respectively

* CDC/AAP case definition

*** Half-reduced CDC/AAP case definition 
Table 4

Accuracy of four best performed PMPE protocols in estimating periodontitis prevalence according to halfreduced CDC/AAP case definition in NHANES sample by age and gender

\begin{tabular}{|c|c|c|c|c|c|c|c|c|}
\hline & \multicolumn{2}{|c|}{ Periodontitis prevalence $(\%)$} & \multicolumn{2}{|c|}{ Absolute bias (\%) } & \multicolumn{2}{|c|}{ Relative bias (\%) } & \multicolumn{2}{|c|}{ Inflation factor (\%) } \\
\hline & Moderate PD (SE) & Severe PD (SE) & Moderate PD & Severe PD & Moderate PD & Severe PD & Moderate PD & Severe PD \\
\hline \multicolumn{9}{|l|}{ Age 30-39 yrs } \\
\hline FMPE $^{*}$ & $15.8(1.2)$ & $3.5(0.6)$ & Ref & Ref & Ref & Ref & Ref & Ref \\
\hline \multicolumn{9}{|l|}{ Full-mouth PMPEs ${ }^{* *}$} \\
\hline CPI & $19.7(1.3)$ & $5.5(0.8)$ & 3.9 & 2.0 & 24.7 & 57.1 & 80.2 & 63.6 \\
\hline MB-DB & $17.8(1.3)$ & $4.3(0.7)$ & 2.0 & 0.8 & 12.7 & 22.9 & 88.8 & 81.4 \\
\hline MB-DL & $18.5(1.3)$ & $4.9(0.7)$ & 2.7 & 1.4 & 17.1 & 40.0 & 85.4 & 71.4 \\
\hline Half-mouth 4 sites ${ }^{* *}$ & $17.3(1.3)$ & $4.3(0.7)$ & 1.5 & 0.8 & 9.5 & 22.9 & 91.3 & 81.4 \\
\hline \multicolumn{9}{|l|}{ Age 40-49 yrs } \\
\hline FMPE $^{*}$ & $26.2(1.5)$ & $10.3(1.0)$ & Ref & Ref & Ref & Ref & Ref & Ref \\
\hline \multicolumn{9}{|l|}{ Full-mouth PMPEs ${ }^{* *}$} \\
\hline CPI & $33.3(1.6)$ & $9.7(1.0)$ & 7.1 & -0.6 & 27.1 & -5.8 & 78.7 & 106.2 \\
\hline MB-DB & $34.0(1.6)$ & $8.2(0.9)$ & 7.8 & -2.1 & 29.8 & -20.4 & 77.1 & 125.6 \\
\hline MB-DL & $32.2(1.6)$ & $11.0(1.0)$ & 6.0 & 0.7 & 22.9 & 6.8 & 81.4 & 93.6 \\
\hline Half-mouth 4 sites ${ }^{* *}$ & $31.0(1.5)$ & $8.6(0.9)$ & 4.8 & -1.7 & 18.3 & -16.5 & 84.5 & 119.8 \\
\hline \multicolumn{9}{|l|}{ Age 50-59 yrs } \\
\hline FMPE $^{*}$ & $39.7(1.8)$ & $18.1(1.4)$ & Ref & Ref & Ref & Ref & Ref & Ref \\
\hline \multicolumn{9}{|l|}{ Full-mouth PMPEs ${ }^{* *}$} \\
\hline CPI & $43.8(1.8)$ & $17.4(1.4)$ & 4.1 & -0.7 & 10.3 & -3.9 & 90.6 & 104.0 \\
\hline MB-DB & $45.9(1.8)$ & $15.6(1.3)$ & 6.2 & -2.5 & 15.6 & -13.8 & 86.5 & 116.0 \\
\hline MB-DL & $44.8(1.8)$ & $19.0(1.5)$ & 5.1 & 0.9 & 12.8 & 5.0 & 88.6 & 95.3 \\
\hline Half-mouth 4 sites ${ }^{* *}$ & $40.5(1.8)$ & $17.0(1.4)$ & 0.8 & -1.1 & -2.0 & -6.1 & 98.0 & 106.5 \\
\hline \multicolumn{9}{|l|}{ Age 60-69 yrs } \\
\hline FMPE ${ }^{*}$ & $45.6(2.0)$ & $17.2(1.5)$ & Ref & Ref & Ref & Ref & Ref & Ref \\
\hline \multicolumn{9}{|l|}{ Full-mouth PMPEs ${ }^{* *}$} \\
\hline CPI & $46.9(2.0)$ & $15.6(1.4)$ & 1.3 & -1.6 & 2.9 & -9.3 & 97.2 & 110.3 \\
\hline MB-DB & $51.2(2.0)$ & $15.1(1.4)$ & 5.6 & -2.1 & 12.3 & -12.2 & 89.1 & 113.9 \\
\hline MB-DL & $51.2(2.0)$ & $17.3(1.5)$ & 5.6 & 0.1 & 12.3 & 0.6 & 89.1 & 99.4 \\
\hline Half-mouth 4 sites ${ }^{* *}$ & $49.4(2.0)$ & $15.4(1.4)$ & 3.8 & -1.8 & 8.3 & -10.5 & 92.3 & 111.7 \\
\hline \multicolumn{9}{|l|}{ Age 70-80 yrs } \\
\hline FMPE $^{*}$ & $57.8(2.2)$ & $12.9(1.5)$ & Ref & Ref & Ref & Ref & Ref & Ref \\
\hline \multicolumn{9}{|l|}{ Full-mouth PMPEs ${ }^{* *}$} \\
\hline CPI & $58.3(2.1)$ & $11.7(1.4)$ & -0.5 & -1.2 & -0.9 & -9.3 & 99.1 & 110.3 \\
\hline MB-DB & $62.3(2.1)$ & $10.2(1.3)$ & 4.5 & -2.7 & 7.8 & -20.9 & 92.8 & 126.5 \\
\hline
\end{tabular}




\begin{tabular}{|c|c|c|c|c|c|c|c|c|}
\hline & \multicolumn{2}{|c|}{ Periodontitis prevalence $(\%)$} & \multicolumn{2}{|c|}{ Absolute bias (\%) } & \multicolumn{2}{|c|}{ Relative bias (\%) } & \multicolumn{2}{|c|}{ Inflation factor (\%) } \\
\hline & Moderate PD (SE) & Severe PD (SE) & Moderate PD & Severe PD & Moderate PD & Severe PD & Moderate PD & Severe PD \\
\hline MB-DL & $62.9(2.1)$ & $12.3(1.4)$ & 5.1 & -0.6 & 8.8 & -4.7 & 91.9 & 104.9 \\
\hline Half-mouth 4 sites ${ }^{* *}$ & $63.4(2.1)$ & $10.0(1.3)$ & 5.6 & -2.9 & 9.7 & -22.5 & 91.2 & 129.0 \\
\hline \multicolumn{9}{|l|}{ Male } \\
\hline FMPE $^{*}$ & $38.8(1.1)$ & $16.7(0.9)$ & Ref & Ref & Ref & Ref & Ref & Ref \\
\hline \multicolumn{9}{|l|}{ Full-mouth PMPEs ${ }^{* *}$} \\
\hline CPI & $43.4(1.2)$ & $16.6(0.9)$ & 4.6 & -0.1 & 11.9 & -0.6 & 89.4 & 100.6 \\
\hline MB-DB & $45.6(1.2)$ & $14.6(0.8)$ & 6.8 & -2.1 & 17.5 & -12.6 & 85.1 & 114.4 \\
\hline MB-DL & $43.9(1.2)$ & $17.9(0.9)$ & 5.1 & 1.2 & 13.1 & 7.2 & 88.4 & 93.3 \\
\hline Half-mouth 4 sites ${ }^{* *}$ & $41.9(1.2)$ & $15.6(0.8)$ & 3.1 & -1.1 & 8.0 & -6.6 & 92.6 & 107.1 \\
\hline \multicolumn{9}{|l|}{ Female } \\
\hline FMPE $^{*}$ & $29.8(1.1)$ & $6.9(0.6)$ & Ref & Ref & Ref & Ref & Ref & Ref \\
\hline \multicolumn{9}{|l|}{ Full-mouth PMPEs ${ }^{* *}$} \\
\hline CPI & $32.8(1.1)$ & $6.5(0.6)$ & 3.0 & -0.4 & 10.1 & -5.8 & 90.9 & 106.2 \\
\hline MB-DB & $33.4(1.1)$ & $5.8(0.5)$ & 3.6 & -1.1 & 12.1 & -15.9 & 89.2 & 119.0 \\
\hline MB-DL & $34.3(1.1)$ & $7.0(0.6)$ & 4.5 & 0.1 & 15.1 & 1.4 & 86.9 & 98.6 \\
\hline Half-mouth 4 sites ${ }^{* *}$ & $33.1(1.1)$ & $5.7(0.5)$ & 3.3 & -1.2 & 11.1 & -17.4 & 90.0 & 121.1 \\
\hline
\end{tabular}

$\mathrm{PD}=$ periodontitis

Negative and positive biases mean underestimation and overestimation of periodontitis prevalence, respectively

* CDC/AAP case definition

*** Half-reduced CDC/AAP case definition 